\title{
Is Neutrophil-to-Lymphocyte Ratio a Prediction Marker for Success of Immunotherapy? Preliminary Results
}

\author{
Noam Asna ${ }^{1}{ }^{*}$, Ornit Cohen ${ }^{2 *}$, Ron Batash ${ }^{3 \#, ~ A s a f ~ S i g r o n}{ }^{1}$, Tatiana Harkovski1 ${ }^{1}$ Moshe Schaffer ${ }^{1,4}$ \\ ${ }^{1}$ Department of Oncology, Barzilai Medical Center, Ashkelon, Israel \\ ${ }^{2}$ Research Center, Barzilai Medical Center, Ashkelon, Israel \\ ${ }^{3}$ Orthopedic Department, Barzilai Medical Center, Ashkelon, Israel \\ ${ }^{4}$ Faculty of Medicine, Ben Gurion University of the Negev, Beer Sheva, Israel \\ Email:ron.batash@gmail.com
}

How to cite this paper: Asna, N., Cohen, O., Batash, R., Sigron, A., Harkovski, T. and Schaffer, M. (2018) Is Neutrophil-to-Lymphocyte Ratio a Prediction Marker for Success of Immunotherapy? Preliminary Results. Open Journal of Immunology, 8, 29-35.

https://doi.org/10.4236/oji.2018.82003

Received: April 2, 2018

Accepted: June 26, 2018

Published: June 29, 2018

Copyright (C) 2018 by author and Scientific Research Publishing Inc. This work is licensed under the Creative Commons Attribution International License (CC BY 4.0).

http://creativecommons.org/licenses/by/4.0/

\section{(c) (i) Open Access}

\begin{abstract}
Background: Cancer immunotherapy treatments enhance the cancer treatment approach. The neutrophil-to-lymphocyteratio (NLR) has been associated with cancer prognosis, affecting progression, chemosensitivity, radiosensitivity and toxicity. Patients and Methods: Ten patients (nine males, one female), aged $68 \pm 10$ years, were included in this study. Six patients had squamous cell carcinoma of the lungs and two had adenocarcinoma of the lungs. One patient suffered from limb soft tissue sarcoma and one had renal cell carcinoma. All patients had metastatic disease and were pretreated with chemotherapy or biologic agents. The rationale of testing NLR was to compare it to the clinical response of the patients assessed by RECIST criteria using computerized tomography (CT) scans. Results: In regression analysis, older patients were found to respond better to immunotherapy treatment than younger patients. NLR was $4.26 \pm 2.25(\mathrm{p}<0.01)$ in the older patients group and $2.84 \pm 0.90(\mathrm{p}<0.01)$ in the younger patients group. No difference in response according to tumor type $(\mathrm{p}=0.479)$ was observed. Conclusion: We provide preliminary evidence demonstrating that low NLR helps in understanding the value of the underlying immune system in expecting a good outcome to immune treatment. The NLR is a simple and available biomarker, easy to apply in clinical practice.
\end{abstract}

\section{Keywords}

Neutrophil-to-Lymphocyteratio (NLR), Cancer Immunotherapy, Biomarker, Cancer Treatment, Chemotherapy

${ }^{\star}$ These authors contributed equally to this paper.

${ }^{\#}$ Corresponding author. 


\section{Introduction}

Cancer immunotherapy treatments enhance the innate powers of the immune system to fight cancer and represent the most promising new cancer treatment approach since the development of the first chemotherapies in the late 1940s. Immunology has led to major treatment revolutions for several cancers types [1] [2]. The innate immune system acts as a first line of defense including a vast number of components, while the adaptive immune response results in the development of cytotoxic $\mathrm{CD}^{+} \mathrm{T}$-cells, helper $\mathrm{CD}^{+} \mathrm{T}$-cells, and antibody-producing plasma cells [3] [4]. The adaptive immune system contains several highly specific antigen receptors on $\mathrm{T}$ - and B-cells, and the binding of an antigen to the $\mathrm{B}$ - or $\mathrm{T}$-cell receptor promotes the progress of an antigen-specific immune response and the growth of long-lived memory cells. After the suppression of cancer, the memory cells potentially prevent tumor regrowth, reduce metastatic spread, and limit de novo initiation of secondary malignancy [5].

Evasion mechanisms used by cancer cells in order to escape the host immune response are important targets for immunotherapy. The loss of antigen expressions along with cytotoxicity resistance, demonstrate how tumor cells overcome immunity. Tumor cells can actively induce an immunosuppressive state. With the ultimate aim of remodeling the tumor microenvironment, some immunotherapeutic approaches to inhibit tumor-induced immunosuppression have been introduced. PD-1, an inhibitory receptor located on T-cells and B-cells surface, binds to programmed death-ligands 1 and 2 (PD-L1 and PD-L2). PD-L1 is widely expressed on hematopoietic and non-hematopoietic cells. The main part of the PD-1/PD-L1 system is to limit the effector T-cells response and immune-mediated tissue damage [6]. In Particular, the use of mAbs directed at PD-1, PD-L1, and CTLA-4 has presented promising outcomes [6]. Nivolumab is an IgG4 $\mathrm{mAb}$ that targets $\mathrm{PD}-1$. This $\mathrm{mAb}$ has shown anti-cancer activity versus several tumor types including non small cell lung cancer (NSCLC) [3] [4] [5]. The first Phase I clinical trial of an anti-PD-1 antibody revealed activity in NSCLC, and subsequent studies have demonstrated that a PD-1 pathway blockade enables durable tumor responses [7]. Completed Phase I and II clinical trials resulted in the FDA approval of second-line chemotherapy regimens for resistant squamous NSCLC.

The safety of nivolumab in NSCLC patients, specially in those with squamous cell cancer, was established in a Phase II trial (CHECKMATE-063) in patients who had progressed after systemic chemotherapy. The most common adverse events (AEs) were fatigue (50\%), dyspnea (38\%), musculoskeletal pain (36\%), decreased appetite (35\%), cough (32\%), nausea (29\%), and constipation (24\%). Serious AEs occurred in 59\% of patients including dyspnea, pneumonia, an exacerbation of COPD, pneumonitis, pleural effusion, and hemoptysis. These adverse reactions led to the termination of treatment in $27 \%$ of patients [8].

In one randomized trial, patients received mono-therapeutic nivolumab (3 $\mathrm{mg} / \mathrm{kg}$ every 2 weeks, intravenously) or docetaxel $\left(75 \mathrm{mg} / \mathrm{m}^{2}\right.$ every 3 weeks, intravenously) displayed that the median overall survival (OS) was higher in pa- 
tients in the nivolumab group than in the docetaxel group (9.2 versus 6.0 months) (95\% CI 7.3 - 13.3) (hazard ratio [HR] 0.59, 95\% CI $0.44-0.79 ; \mathrm{p}<$ 0.001). At 1 year, the OS rate was $42 \%$ (95\% CI $34-50)$ in the nivolumab group and $24 \%(95 \%$ CI $17-31)$ in the docetaxel group. Nine of $131(7 \%)$ patients in the nivolumab group and 71 (55\%) of 129 in the docetaxel group had grade 3/4 treatment-related AEs [9].

The effective use of nivolumab as a second-line therapy led to studies of nivolumab as a first-line treatment. Presently, nivolumab is being studied in a Phase I trial to evaluate its safety and tolerability as a first-line combination- or mono-therapy in chemotherapy-naïve patients [10].

The neutrophil-to-lymphocyte ratio (NLR) has been associated with cancer prognosis, influencing progression, chemosensitivity, radiosensitivity, and toxicity, while the distribution of NLR in healthy population was not formally studied. In healthy populations, Results demonstrated that NLR was positively associated with age. The eldest age group possessed the highest NLR and the youngest age group had the lowest NLR [11]. If elevated, worse outcomes in several malignancies were revealed [12] [13] [14] [15]. The role of NLR in immune therapy is not well defined, and only scarce information is available [16] [17]. In this paper we present our first observation of NLR in patients treated with immunotherapy as at least second line treatment.

\section{Patients and Methods}

A cross-sectional study of 10 patients included nine males and one female, aged $68 \pm 10$ years. Six patients had squamous cell carcinoma of the lung and two had adenocarcinoma of the lung. One patient suffered from soft tissue sarcoma of the limb and one patient had renal cell carcinoma. All patients had metastatic disease and were pretreated with chemotherapy or biologic agents (see Table 1 sample characteristics frequencies).

Table 1. Sample characteristics frequencies.

\begin{tabular}{|c|c|c|c|c|}
\hline & Characteristics & $\begin{array}{l}\text { No reaction } \\
(\%, n=4)\end{array}$ & $\begin{array}{c}\text { Reaction } \\
(\%, n=6)\end{array}$ & $P$ value \\
\hline \multirow{12}{*}{ Diagnosis } & Male $(\%), \mathrm{n}=9 / 10$ & $33 \%, n=3$ & $67 \%, \mathrm{n}=6$ & 0.197 \\
\hline & Adeno Crcinoma of Lung & 0 & $33 \%, \mathrm{n}=2$ & \\
\hline & SCC of Lung & $75 \%, \mathrm{n}=3$ & $50 \%, \mathrm{n}=3$ & 0.256 \\
\hline & Renal cell Carcinoma & $25 \%, \mathrm{n}=1$ & 0 & \\
\hline & \multirow[t]{2}{*}{ Soft Tissue Sarcoma } & & $16.7 \%, \mathrm{n}=1$ & \\
\hline & & $\begin{array}{c}\text { No reaction } \\
(\%, n=4)\end{array}$ & $\begin{array}{l}\text { Reaction } \\
(\%, n=6)\end{array}$ & $P$ value \\
\hline & Age & $59 \pm 6$ & $74 \pm 7$ & ${ }^{\star} 0.001$ \\
\hline & Days & $15.6 \pm 3$ & $16 \pm 7$ & 0.805 \\
\hline & Leucocytes & $7.9 \pm 1.78$ & $7.64 \pm 1.98$ & 0.353 \\
\hline & Neutrophils & $5.42 \pm 1.62$ & $4.73 \pm 1.51$ & ${ }^{\star} 0.025$ \\
\hline & Lymphocytes & $1.45 \pm 0.49$ & $1.78 \pm 0.63$ & $\star 0.006$ \\
\hline & NLR & $4.27 \pm 2.26$ & $2.85 \pm 0.9$ & ${ }^{\star} 0.001$ \\
\hline
\end{tabular}


The ratio of NLR was compared to the clinical response of the patients assessed by RECIST criteria using computer computerized tomography (CT) scans [18]. The evaluation of NLR was done after 12 measurements of neutrophils and leucocytes in each patient taken before each cycle of therapy and occasionally between treatments if the patient was hospitalized or had arrived at the clinic, Median treatment time was 16 days (range, 14 - 63 days), and immunotherapy is given once every two or three weeks.

Inclusion criteria were any patients older than 18 years old, with advanced cancer treated with chemotherapy and biological therapy, without success and was indicated to immune treatment. Exclusion criteria: Patients under 18 years old, pregnancy women, and patients that did not received chemotherapy as first line treatment.

The study protocol conformed to the ethical guidelines according to the Declaration of Helsinki and ICH-GCP as reflected a priori after approval by the institution's Helsinki committee (0026-17-BRZ).

\section{Statistics}

First, a univariate analysis was performed to all variables to identify parameters of patients who responded to treatment with PD-1 inhibitors. Chi-square statistics were used for categorical variables and one way ANOVA was used for numerical variables. After ruling out multi-co-linear relationship, logistic regression was used to distinguish independent predictors of patients who responded to PD-1 inhibitors versus patients with no response. Since all independent variables in this model were categorical, exponential coefficient estimates may be interpreted as conditional odds ratios. All comparisons were exploratory. Pvalues account statistical significance (two sided, alpha level 5\%). Analyses were performed using IBM-SPSS vs. 24.

\section{Results}

Median treatment time was 16 days (range, 14 - 63 days), and immunotherapy is given once every two or three weeks.

In one way ANOVA regression analysis, we found that older patients responded better to PD-1 inhibitors treatment than did younger patients. Older patients had significant lower NLR than younger patients who did not show response. Non-responders were significantly younger than the responders, $59 \pm$ 6.32 years versus $74 \pm 6.58$ years, $\mathrm{p}<0.001$.

The NLR in the younger patients group (under 65 years of age)was significantly higher than that in the older patients group (older than 65 years of age), $4.26 \pm 2.25,(\mathrm{p}<0.01)$ versus $2.84 \pm 0.90(\mathrm{p}<0.01)$. A significant correlation between response (older patients) to treatment was therefore demonstrated $(\mathrm{R}=$ 0.226 , $\mathrm{p}$ value $=0.01)$.

Measurements of neutrophils and leucocytes were found to be $12.30 \pm 5.37$ times (range $2-9, p<0.005$ ). 
A clear interaction was demonstrated using a logistic regression model between NLR and patient age $(B=0.426,95 \%$ CI $1.26-1.86$, $\mathrm{p}$ value $<0.001)$.

Younger patients with a high NLR had less chances of having a clinical response (Figure 1). We did not observe any differences in response according to tumor type $(\mathrm{p}=0.479)$.

\section{Discussion}

The NLR has been associated with cancer prognosis, influencing progression and chemosensitivity, radiosensitivity and toxicity. If elevated, NLR is associated with worse outcomes in several malignancies [12] [13] [14] [15].

The role of NLR in immune therapy is not well defined, and only scarce information is available [16] [17]. In a cohort of 175 patients with NSCLC treated with nivolumab in routine practice, Bagley et al noted that pretreatment NLR $\geq$ 5 was associated with inferior outcomes [16]. It is unclear whether this marker is predictive or prognostic. In our preliminary study, the cohort of patients is small and they had different tumors. However, a large number of measurements was taken for each patient and showed that patients with low NLR responded better to immunotherapy than did patients with high NLR. This is confirmed by Templeton et al who demonstrated that the immune cells proportion in the blood is of prognostic value, hence, a decrease of the proportion of neutrophils to lymphocytes was associated with more positive outcomes, while an increase had the reverse effect [19]. A different effect is known in chemotherapy and in radiation treatment; patients who presented with higher NLRs and lower serum albumin levels experienced less treatment-related symptomatic toxicity [12] [13] [14] [15] [20]. In our preliminary finding we found a difference of age in correlation to NLR, where the oldest patients had the lowest NLR and better response. We have no explanation for this phenomenon. To the best of our knowledge, no similar report has been found in the literature.

We have provided initial evidence, based on a relatively high number of measurements of neutrophils and leucocytes $(12.30 \pm 5.37)$, that a low NLR helps

Ratio neutrophils/lymphocytes

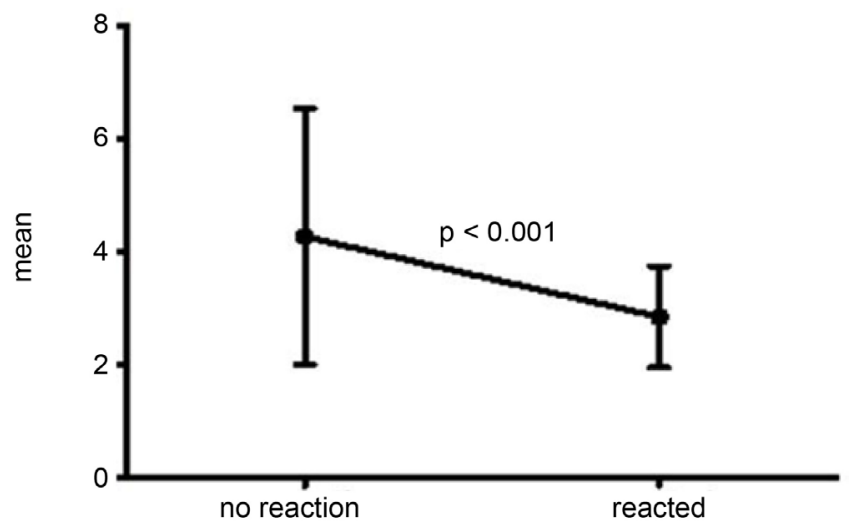

Figure 1. Ratio of neutrophils to lymphocytes. 
probably, to understand the value of the underlying immune system in predicting a good outcome to immune treatment. However, the parameter has a potential to be a marker with further systemic study with more homogeneous sample to appreciate the results.

\section{Conclusion}

The neutrophil-to-lymphocyte ratio is an accessible biomarker, easy to implement in common practice. The NLR can be used in addition to the other established prognostic factors and newer genomic predictors; the NLR could perfect therapeutic algorithms and assist in decision-making in regards with the need for immune therapy, currently used in cancer patients. In conclusion, the NLR is an easy, not complicated and cheap prognostic factor, that can improve the various immune treatments in oncology.

\section{Conflicts of Interest}

The authors report no conflicts of interest.

\section{Contributors' List}

NA, OC: initiators, RB reviewed and corrected, MS: performed statistical calculations, analyzed data; AS: collected data; TH: reviewed and corrected the MS and analyzed the data; MS supervised and analyzed the data.

\section{References}

[1] Pasquali, S., Chiarion-Sileni, V., Rossi, C.R. and Mocellin, S. (2017) Immune Checkpoint Inhibitors and Targeted Therapies for Metastatic Melanoma: A Network Meta-Analysis. Cancer Treatment Reviews, 54, 34-42. https://doi.org/10.1016/j.ctrv.2017.01.006

[2] de Mello, R.A., Veloso, A.F., Esrom Catarina, P., Nadine, S. and Antoniou, G. (2016) Potential Role of Immunotherapy in Advanced Non-Small-Cell Lung Cancer. OncoTargets and Therapy, 10, 21-30. https://doi.org/10.2147/OTT.S90459

[3] Madureira, P., de Mello, R.A., de Vasconcelos, A. and Zhang, Y. (2015) Immunotherapy for Lung Cancer: For Whom the Bell Tolls? Tumor Biology, 36, 1411-1422. https://doi.org/10.1007/s13277-015-3285-6

[4] Ernst, B. and Anderson, K.S. (2015) Immunotherapy for the Treatment of Breast Cancer. Current Oncology Reports, 17, 5.

https://doi.org/10.1007/s11912-014-0426-9

[5] Page, D.B., Bourla, A.B., Daniyan, A., Naidoo, J., Smith, E., Smith, M., et al. (2015) Tumor Immunology and Cancer Immunotherapy: Summary of the 2014 SITC Primer. Journal for ImmunoTherapy of Cancer, 3, 25. https://doi.org/10.1186/s40425-015-0072-2

[6] Vesely, M.D., Kershaw, M.H., Schreiber, R.D. and Smyth, M.J. (2011) Natural Innate and Adaptive Immunity to Cancer. Annual Review of Immunology, 29, 235-271. https://doi.org/10.1146/annurev-immunol-031210-101324

[7] Ang, Y.L.E., Tan, H.-L. and Soo, R.A. (2015) Best Practice in the Treatment of Advanced Squamous Cell Lung Cancer. Therapeutic Advances in Respiratory Disease, 9, 224-235. https://doi.org/10.1177/1753465815581147 
[8] Rizvi, N.A., Mazières, J., Planchard, D., Stinchcombe, T.E., Dy, G.K., Antonia, S.J., et al. (2015) Activity and Safety of Nivolumab, an Anti-PD-1 Immune Checkpoint Inhibitor, for Patients with Advanced, Refractory Squamous Non-Small-Cell Lung Cancer (CheckMate 063): A Phase 2, Single-Arm Trial. The Lancet Oncology, 16, 257-265. https://doi.org/10.1016/S1470-2045(15)70054-9

[9] Yaqub, F. (2015) Nivolumab for Squamous-Cell Non-Small-Cell Lung Cancer. The Lancet Oncology, 16, e319. https://doi.org/10.1016/S1470-2045(15)00033-9

[10] Rounds, A. and Kolesar, J. (2015) Nivolumab for Second-Line Treatment of Metastatic Squamous Non-Small-Cell Lung Cancer. American Journal of Health-System Pharmacy, 72, 1851-1855. https://doi.org/10.2146/ajhp150235

[11] Li, J., Chen, Q., Luo, X., Hong, J., Pan, K., Lin, X., Liu, X., Zhou, L., Wang, H., Xu, Y., Li, H. and Duan, C. (2015) Neutrophil-to-Lymphocyte Ratio Positively Correlates to Age in Healthy Population. Journal of Clinical Laboratory Analysis, 29, 437-443. https://doi.org/10.1002/jcla.21791

[12] Buisan, O., Orsola, A., Areal, J., Font, A., Oliveira, M., Martinez, R. and Ibarz, L. (2017) Low Pretreatment Neutrophil-to-Lymphocyte Ratio Predicts for Good Outcomes in Patients Receiving Neoadjuvant Chemotherapy before Radical Cystectomy for Muscle Invasive Bladder Cancer. Clinical Genitourinary Cancer, 15, 145-151.e2. https://doi.org/10.1016/j.clgc.2016.05.004

[13] Boissier, R., Campagna, J., Branger, N., Karsenty, G. and Lechevallier, E. (2017) The Prognostic Value of the Neutrophil-Lymphocyte Ratio in Renal Oncology: A Review. Urologic Oncology, 35, 135-141. https://doi.org/10.1016/j.urolonc.2017.01.016

[14] Personeni, N., Giordano, L., Abbadessa, G., Porta, C., Borbath, I., Daniele, B., et al. (2017) Prognostic Value of the Neutrophil-to-Lymphocyte Ratio in the ARQ 197-215 Second-Line Study for Advanced Hepatocellular Carcinoma. Oncotarget, 8, 14408-14415. https://doi.org/10.18632/oncotarget.14797

[15] Shaverdian, N., Veruttipong, D., Wang, J., Schaue, D., Kupelian, P. and Lee, P. (2016) Pretreatment Immune Parameters Predict for Overall Survival and Toxicity in Early-Stage Non-Small-Cell Lung Cancer Patients Treated with Stereotactic Body Radiation Therapy. Clinical Lung Cancer, 17, 39-46. https://doi.org/10.1016/j.cllc.2015.07.007

[16] Bagley, S.J., Kothari, S., Aggarwal, C., Bauml, J.M., Alley, E.W., Evans, T.L., et al. (2017) Pretreatment Neutrophil-to-Lymphocyte Ratio as a Marker of Outcomes in Nivolumab-Treated Patients with Advanced Non-Small-Cell Lung Cancer. Lung Cancer, 106, 1-7. https://doi.org/10.1016/j.lungcan.2017.01.013

[17] Nakamura, Y., Kitano, S., Takahashi, A., Tsutsumida, A., Namikawa, K., Tanese, K., et al. (2016) Nivolumab for Advanced Melanoma: Pretreatment Prognostic Factors and Early Outcome Markers during Therapy. Oncotarget, 7, 77404-77415. https://doi.org/10.18632/oncotarget.12677

[18] Feinberg, B.A., Bharmal, M., Klink, A.J., Nabhan, C. and Phatak, H. (2018) Using Response Evaluation Criteria in Solid Tumors in Real-World Evidence Cancer Research. Future Oncology. https://doi.org/10.2217/fon-2018-0317

[19] Templeton, A.J., Knox, J.J., Lin, X., Simantov, R., Xie, W., Lawrence, N., et al. (2016) Change in Neutrophil-to-Lymphocyte Ratio in Response to Targeted Therapy for Metastatic Renal Cell Carcinoma as a Prognosticator and Biomarker of Efficacy. European Urology, 70, 358-364. https://doi.org/10.1016/j.eururo.2016.02.033

[20] Chen, J., Hong, D., Zhai, Y. and Shen, P. (2015) Meta-Analysis of Associations between Neutrophil-to-Lymphocyte Ratio and Prognosis of Gastric Cancer. World Journal of Surgical Oncology, 13, 122. https://doi.org/10.1186/s12957-015-0530-9 